Article

\title{
Application of Extractive Fermentation on the Recuperation of Exopolysaccharide from Rhodotorula mucilaginosa UANL-001L
}

\author{
Carlo Franco Medina-Ramirez ${ }^{1,2}$ (D), Mariana Teresa Castañeda-Guel ${ }^{1,2}$, \\ Ma. Fernanda Alvarez-Gonzalez ${ }^{1,2}$, Alejandro Montesinos-Castellanos ${ }^{3}$, \\ Jose Ruben Morones-Ramirez ${ }^{1,2}$ (D) Enrique A. López-Guajardo ${ }^{3}$ (D) and \\ Alma Gómez-Loredo ${ }^{1,2, *(1)}$ \\ 1 Facultad de Ciencias Químicas, Universidad Autónoma de Nuevo León, Pedro de Alba, S/N, \\ San Nicolas de los Garza, Nuevo León, Mexico; francomedina000@gmail.com (C.F.M.-R.); \\ teremarian@hotmail.com (M.T.C.-G.); mafer01agzz@gmail.com (M.F.A.-G.); \\ jose.moronesrmr@uanl.edu.mx (J.R.M.-R.) \\ 2 Centro de Investigacion en Biotecnologia y Nanotoxicologia, Facultad de Ciencias Quimicas, \\ Universidad Autonoma de Nuevo Leon, Parque de Investigacion e Innovacion Tecnologica, \\ Km. 10 autopista al Aeropuerto Internacional Mariano Escobedo, Apodaca 66629, Nuevo Leon, Mexico \\ 3 Tecnologico de Monterrey, School of Engineering and Sciences, Ave. Eugenio Garza Sada 2501, \\ Monterrey 64849, Nuevo Leon, Mexico; alejandro_montesinos@tec.mx (A.M.-C.); \\ enrique.alopezg@tec.mx (E.A.L.-G.) \\ * Correspondence: alma.gomezlr@uanl.edu.mx
}

Received: 9 September 2020; Accepted: 9 October 2020; Published: 13 November 2020

check for updates

\begin{abstract}
Exopolysaccharides (EPS) are high molecular weight biomaterials of industrial interest due to their variety of applications in the pharmaceutical, cosmetic, environmental, and food industries. EPS produced by Rhodotorula mucilaginosa UANL-001 L has sparked interest due to its bio-adsorbent and wide spectrum antimicrobial properties. However, full exploitation and commercial application of EPS has been restrained due to low yields and high production costs. In the present work, the production and separation of EPS from Rhodotorula mucilaginosa UANL-001L was attempted through extractive fermentation in order to increase EPS production while simplifying the recovery process. Extractive fermentation was implemented with a thermoseparating polymer for phase formation (EOPO 970 and EOPO 12,000); culture viability, biomass generation, EPS production, rheological system properties, and phase formation time and temperature were monitored throughout the process. Extractive fermentation of Rhodotorula mucilaginosa UANL-001L with EOPO 970 resulted in a $42 \%$ EPS and $7 \%$ biomass recovery on the top phase after 5 to 13 -min phase formation time and temperatures between 30 and $40{ }^{\circ} \mathrm{C}$. This is the first report of extractive fermentation application for EPS production by yeast of the genera Rhodotorula, resulting in an interesting strategy for EPS production and recovery, although further optimization is needed.
\end{abstract}

Keywords: extractive fermentation; exopolsaccharide; Rhodotorula mucilaginosa UANL-001L

\section{Introduction}

Exopolysaccharides (EPS) is a high molecular weight biomaterial excreted by various microorganisms [1], especially by the yeast of the following genus: Aureobasidium, Bullera, Candida, Cryptococcus, Pichia, Phomopsis, Exophiala, Lipomyces, Rhodotorula, Sporobolomyces, Tremella, and Trichosporon [2]. Various EPS are of industrial interest due to its variety of applications in pharmaceutical, cosmetic, environmental, and food industries [3], where some EPS, including xanthan, dextran, and hyaluronate, 
are commercially produced [2]. EPS produced by Rhodotorula mucilaginosa UANL-001L has sparked interest due to its bioadsorbent [4] and wide spectrum antimicrobial [5] properties.

However, full exploitation and commercial application of EPS has been restrained due to low yields and high production costs. Several studies on cheaper fermentation substrates, fermentation process optimization, and even genetic engineering have surfaced in order to obtain higher titers [6]. Frequently, the recovery and purification of EPS starts with cell removal by centrifugation or filtration, followed by precipitation with solvents (ethanol, acetone, or isopropanol) and contaminants are removed by enzymatic or physical deproteinization [7]. Nevertheless, recuperation and purification of EPS has become an economical and technical challenge due to the application of unit operations such as centrifugation, sedimentation, and solvent extraction, where recoveries are reported to be not optimal [8].

Consequently, the implementation of effective fermentation and recuperation processes are highly needed. An alternative process is the implementation of extractive fermentation, where fermentation and separation of extracellular products takes place on the same environment at the same time [9]. Extractive fermentation consists in a fermentation process that takes place in an aqueous two-phase system, where the microorganism is able to reproduce on the bottom phase while the extracellular components can be extracted to the top phase. Moreover, extractive fermentation has the potential to increase production and recuperation of metabolites, increasing the overall productivity of the bioprocess $[9,10]$. Furthermore, extractive fermentation can be implemented with thermoseparating agents that allow phase formation depending on the system's temperature, being ethylene oxide and propylene oxide (EOPO) random copolymers the thermoseparating agents most widely used [11]. Then, the extracellular product can be retrieved from the system without the need of separation of solid-liquid unit operations, and without interrupting the fermentation process [9].

In the present work, the production and separation of EPS from Rhodotorula mucilaginosa UANL-001L was attempted through extractive fermentation in order to increase EPS production while simplifying the recovery process. In order to achieve this goal, two main strategies were implemented. First, media composition (Yeast Malt Broth (YM) medium vs mineral medium) was studied to determine if EPS production on submerged fermentation was affected. Then, extractive fermentation was implemented with a thermoseparating polymer for phase formation (EOPO 970 and EOPO 12,000), monitoring culture viability, biomass generation, and EPS production. Finally, three factors such as phase formation time, temperature and EOPO concentration were studied for the thermoseparating polymer that yielded the highest biomass and EPS production, in terms of EPS and biomass recovery on each phase.

\section{Materials and Methods}

\subsection{Media Composition Effect on EPS Production}

\subsubsection{Cultivation Procedures in Submerged Fermentation}

Rhodotorula mucilaginosa UANL-001 L was provided on solid medium by Laboratorio de Biología Sintética y de Sistemas at CIBYN, México. In order to work with liquid cultures, a colony from the plate was taken with an inoculation loop, inoculated on $50 \mathrm{~mL}$ Yeast Malt Broth (YM) medium (Sigma Aldrich, Toluca, México) on sterile conditions, and kept in incubation for 3 days at $30^{\circ} \mathrm{C}$ and $250 \mathrm{rpm}$ (standard conditions). Later, $10 \mathrm{~mL}$ of culture were transferred to fresh YM medium $(100 \mathrm{~mL})$ to obtain the starting culture. YM medium composition is shown in Table 1.

Table 1. Yeast Malt Broth (YM) medium composition.

\begin{tabular}{cc}
\hline Component & Concentration $(\mathrm{g} / \mathrm{L})$ \\
\hline Peptic digest of animal tissue & 5.0 \\
Yeast extract & 3.0 \\
Malt extract & 3.0 \\
Dextrose & 10.0 \\
\hline
\end{tabular}


Mineral medium was supplemented with saccharose and prepared according the formulation on Table 2. Cultures with $100 \mathrm{~mL}$ were used for media composition studies. $20 \mathrm{~mL}$ of starting culture were inoculated in both YM and mineral medium and kept for 7 days on standard conditions. Every $24 \mathrm{~h}$, $5 \mathrm{~mL}$ samples were taken for biomass and EPS quantification.

Table 2. Mineral medium composition.

\begin{tabular}{cc}
\hline Component & Concentration $(\mathrm{g} / \mathrm{L})$ \\
\hline Ammonium chloride $\left(\mathrm{NH}_{4} \mathrm{Cl}\right)$ & 2.0 \\
Potassium phosphate $\left(\mathrm{KH}_{2} \mathrm{PO}_{4}\right)$ & 1.0 \\
Magnesium sulphate $\left(\mathrm{MgSO}_{4}\right)$ & 0.5 \\
Sodium chloride $(\mathrm{NaCl})$ & 0.1 \\
Calcium chloride $(\mathrm{CaCl})$ & 0.1 \\
Zinc sulphate $\left(\mathrm{ZnSO}_{4}\right)$ & 0.005 \\
Saccharose $\left(\mathrm{C}_{12} \mathrm{H}_{22} \mathrm{O}_{11}\right)$ & 100.0 \\
\hline
\end{tabular}

\subsubsection{Cultivation Procedures in Presence of EOPO}

For extractive fermentation, EOPO 970 and EOPO 12,000 (Sigma Aldrich, Toluca, México) were used. In order to initiate extractive fermentation, $10 \mathrm{~mL}$ of an starting culture were transferred to $100 \mathrm{~mL}$ of YM or mineral medium with 20 or 30\% $(v / v)$ EOPO 12,000 or 10, 20, 30, 40, or 50\% (v/v) EOPO 970. As a control, $10 \mathrm{~mL}$ of an starting culture on $100 \mathrm{~mL}$ YM or mineral medium without EOPO was used.

\subsubsection{Biomass and EPS Recuperation in Submerged Fermentation}

A $5 \mathrm{~mL}$ sample from the submerged fermentation was retrieved and centrifuged at $9500 \mathrm{rpm}$ for $20 \mathrm{~min}$ at $4{ }^{\circ} \mathrm{C}$. The supernatant was reserved at $50 \mathrm{~mL}$ in falcon tubes and the pellet was reserved at $-20^{\circ} \mathrm{C}$ for further biomass quantification. The supernatant was filtered through a $0.2 \mu \mathrm{m}$ cellulose membrane. Two volumes (5 mL approximately per volume) of $96 \%$ ethanol were added and incubated for $12 \mathrm{~h}$ at $-20{ }^{\circ} \mathrm{C}$ to precipitate EPS. After incubation, the mixture was centrifuged at $9500 \mathrm{rpm}$ for $20 \mathrm{~min}$ at $4{ }^{\circ} \mathrm{C}$, discarding the supernatant and washing the pellet twice with $70 \%$ ethanol. Between washes, the EPS was centrifuged at $9500 \mathrm{rpm}$ for $20 \mathrm{~min}$ at $4{ }^{\circ} \mathrm{C}$, discarding the supernatant. Finally, the resulting pellet was dried by ethanol evaporation at room temperature for $15 \mathrm{~min}$.

Once both biomass and EPS pellets were obtained, the water content was evaporated to determine biomass and EPS dry weight. The drying process was performed in an Integrated Speed Vac (ThermoFisher, Waltham, MA, USA) at $45^{\circ} \mathrm{C}, 5$ Torr pressure for $6 \mathrm{~h}$. After this process, dry weight was determined by difference of the dry weight and the original sample weight.

\subsection{EOPO Effect on Cell Viability and Biomass Generation}

\subsubsection{Cell Viability}

In order to verify if Rhodotorula mucilaginosa UANL-001L viability was maintained when EOPO was added to the medium, a cell viability assay was performed. Cells were stained with a methylene blue solution as stated in Table 3. Briefly, $1 \mathrm{~mL}$ culture sample was mixed with $1 \mathrm{~mL}$ of methylene blue solution. The mixture was incubated for $15 \mathrm{~min}$ at $25^{\circ} \mathrm{C}$ and a $300 \mu \mathrm{L}$ sample was then observed in a Neubauer chamber for cell count. Both stained and not stained cells were counted in order to determine cell viability with Equations (1) and (2).

$$
\begin{gathered}
{[C]=\frac{C C}{C q} \times T Q \times F D \times 10,000} \\
V=\frac{([C]-C A)}{[C]} \times 100
\end{gathered}
$$


where:

CCell concentration

CC: Counted cells

Cq: Counted quadrants

CA: Counted blue cells

TQ: Total quadrants

FD: Dilution Factor

V: Cell viability

Table 3. Methylene blue solution composition.

\begin{tabular}{cc}
\hline Component & Concentration $(\mathrm{g} / \mathrm{L})$ \\
\hline Methylene blue & 0.025 \\
Sodium chloride $(\mathrm{NaCl})$ & 0.90 \\
Calcium chloride $\left(\mathrm{CaCl}_{2}\right)$ & 0.04 \\
Potassium chloride $\left(\mathrm{CaCl}_{2}\right)$ & 0.04 \\
\hline
\end{tabular}

\subsubsection{Biomass Generation}

$100 \mathrm{~mL}$ cultures where prepared as stated in Section 2.1.2. Every $24 \mathrm{~h}$, a $10 \mathrm{~mL}$ sample was retrieved for 7 days. Biomass was determined as previously stated in Section 2.1.3

\subsection{Extractive Fermentation Parameter Study: Rheological Characterization and Phase Formation Time and Temperature}

\subsubsection{Rheological Characterization of Two-Phase Systems}

Extractive fermentation is an aqueous two-phase system (ATPS) that requires the interaction and characterization of each phase; however, the properties of the aqueous two-phase systems used in this study are not reported in the literature. Therefore, the experimental measurement of dynamic viscosity, superficial tension, and interfacial tension were performed by varying the EOPO concentration for each media. Dynamic viscosity measurement was carried out with an Ostwald viscometer CANNON 200 AA58 (Cannon Instrument Company, State College, PA, USA) at $25^{\circ} \mathrm{C}$ using water as a reference liquid. Surface tension and interfacial tension were both measured with a DuNouy tensiometer (CSC Scientific, Fairfax, VA, USA). For surface tension, the measured system consisted of the $\mathrm{EOPO}+$ media with air at $25{ }^{\circ} \mathrm{C}$; while for the interfacial tension measurement, the system was heated at the formation phase temperature to form both phases (top: aqueous phase and bottom: EOPO rich phase). Three measurements were performed resulting in a standard error below 5\%.

\subsubsection{Two-Phase Generation in the Prescence of EOPO 970}

For different concentrations of EOPO 970, time and temperature were monitored in order to determine the conditions to generate two-phase systems. First, $50 \mathrm{~mL}$ starting cultures were prepared and maintained in standard conditions for 5 days. Later, 5 different concentrations of EOPO 970 (10, 20, $30,40$, or $50 \%(v / v))$ were added to cultures. Then, each culture was put in a water bath for culture temperature modification $\left(30,35,40\right.$, and $\left.45^{\circ} \mathrm{C}\right)$, by heating the water on the bath as needed. Once the desired temperature was reached, the phase generation time was recorded at the time the two phases were visibly distinctive. Culture temperature was monitored by an infrared thermometer.

\subsection{Biomass and EPS Production and Recovery on Extractive Fermentation with EOPO 970}

After EOPO 970 concentration determination for phase formation, viability retention and phase formation temperature, the biomass and EPS produced were quantified for the top and bottom phase. 
First, $100 \mathrm{~mL}$ cultures were prepared with 20, 30, 40, or 50\% (v/v) EOPO 970. After phase formation, the top phase was removed with a pipette and treated as described in Section 2.1.3 for biomass and EPS quantification. Likewise, the bottom phase of each culture was treated as described in Section 2.1.3 in order to separate and quantify the biomass and EPS retained on the phase.

\subsection{Statistical Analysis}

Statistical analysis was performed using Microsoft Excel (Redmon, WA, USA). One-way Analysis of Variance (ANOVA) was used to determine significant differences ( $p$-value $<0.05)$ between treatments. All experiments were performed in biological duplicates and the mean is reported with the corresponding standard deviations.

\section{Results}

\subsection{Media Composition Effect on EPS Production}

YM and mineral Medium were compared in terms of biomass and EPS production on submerged fermentation. As shown in Figure 1, biomass (a) and EPS (b) production is similar in both YM and mineral media. After $240 \mathrm{~h}$ of cultivation, biomass concentration was $0.0360 \mathrm{~g} / \mathrm{L}$ in YM medium while a $0.0298 \mathrm{~g} / \mathrm{L}$ biomass concentration was obtained from mineral medium. Moreover, it is important to note that EPS production is very low at the first $72 \mathrm{~h}$ of cultivation, resulting in a higher production after $120 \mathrm{~h}$ of culture in both media, being the highest EPS concentration obtained from YM medium $(0.0052 \mathrm{~g} / \mathrm{L})$. Both biomass and EPS maximum concentration from both media resulted not significantly different and proved to be fairly constant after $168 \mathrm{~h}$ of culture.

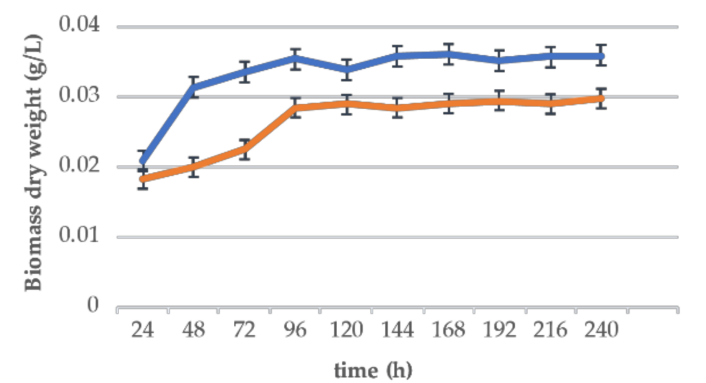

b)

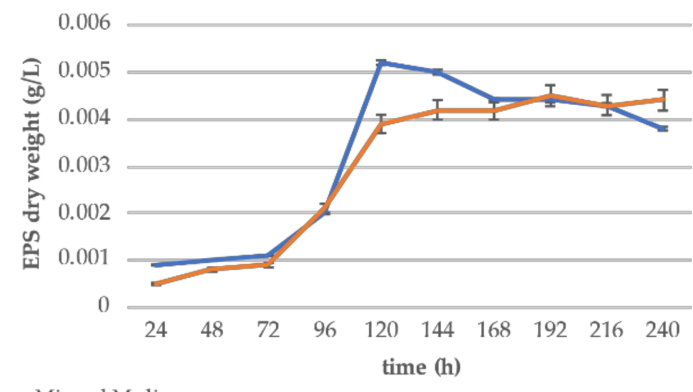

Figure 1. Biomass (a) and EPS (b) production in submerged fermentation of Rhodotorula mucilaginosa UANL-001L cultures in YM and mineral media. Cultures were maintained in the corresponding liquid medium on sterile conditions and kept in incubation for 3 days at $30^{\circ} \mathrm{C}$ and $250 \mathrm{rpm}$.

\subsection{EOPO Effect on Viability and Biomass Generation}

Culture viability in presence of EOPO was evaluated by cell count in a Neubauer chamber and Methylene Blue coloration. Concentrations from $10 \%$ to $50 \%(v / v)$ were tested for EOPO 970, while $20 \%$ and $30 \%(v / v)$ were tested for EOPO 12,000. As shown in Figure 2, cultures in presence of EOPO 12,000 (a) or EOPO 970 (b) showed an important decrease in viability after 96 h. More importantly, culture viability decreased by 35\% after $168 \mathrm{~h}$ in comparison to YM and mineral media controls. Higher EOPO concentrations in both experiments signified higher drops in culture viability, where concentrations higher than 30\% EOPO signified less than 50\% cell viability after $96 \mathrm{~h}$ of culture. 
a)

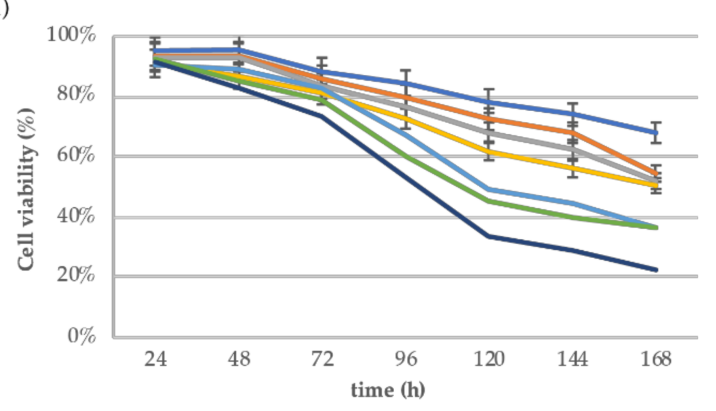

b)

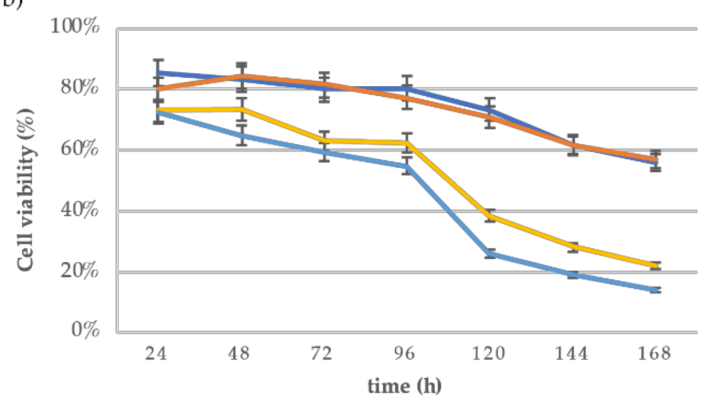

$\longrightarrow$ YM Mineral $-10 \% \mathrm{EOPO}=20 \% \mathrm{EOPO}$ $30 \% \mathrm{EOPO}-40 \% \mathrm{EOPO}-50 \% \mathrm{EOPO}$

Figure 2. Cell viability in submerged fermentation of Rhodotorula mucilaginosa UANL-001L cultures in presence of (a) EOPO 970 or (b) EOPO 12,000. Cultures were maintained in liquid medium on sterile conditions and kept in incubation for 3 days at $30{ }^{\circ} \mathrm{C}$ and $250 \mathrm{rpm}$.

In terms of biomass generation, Figure 3a shows that concentrations of up to $40 \%$ EOPO 970 retrieved similar biomass concentrations as the control after $96 \mathrm{~h}$ of culture. However, biomass concentration in cultures with EOPO 12,000 was significantly lower (about 30\%) than the control, even at early culture stages. Therefore, further studies for extractive fermentation were carried out only with EOPO 970.

a)

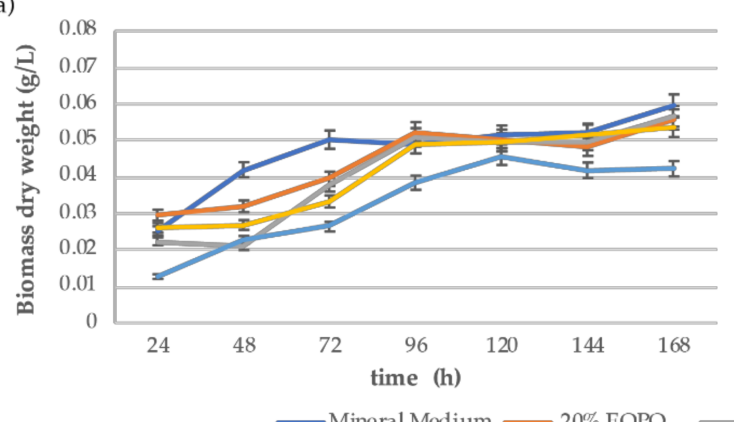

b)

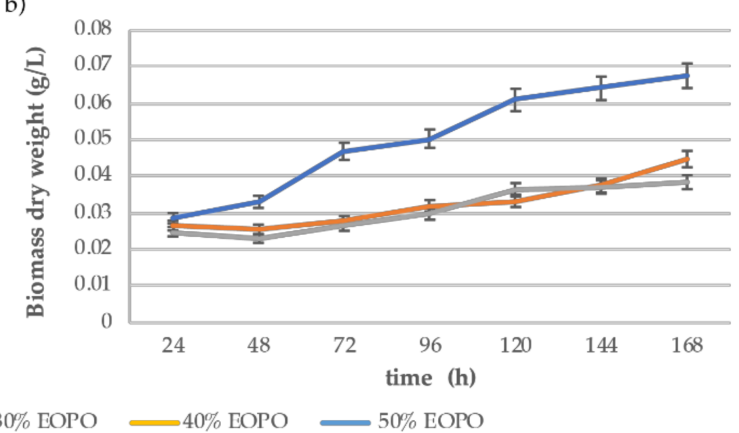

Figure 3. Biomass production in submerged fermentation of Rhodotorula mucilaginosa UANL-001L cultures in presence of (a) EOPO 970 or (b) EOPO 12,000. Cultures were maintained in liquid medium on sterile conditions and kept in incubation for 3 days at $30{ }^{\circ} \mathrm{C}$ and $250 \mathrm{rpm}$.

\subsection{Extractive Fermentation Parameter Study: Rheological Characterization and Phase Formation Time and Temperature}

\subsubsection{Rheological Characterization of Two-Phase Systems}

Experimental measurements of dynamic viscosity, superficial tension, and interfacial tension of the systems, with and without Rhodotorula mucilaginosa UANL-001L, at various EOPO concentrations are presented in Table 4. These results are highly valuable since no data for this system is available in the literature, and extractive fermentation depends on the analysis of the two-phase system.

Viscosity linearly increases with increasing EOPO concentrations in both media, with and without the presence of Rhodotorula mucilaginosa UANL-001L. More importantly, media viscosity increases about $133 \%$ when Rhodotorula mucilaginosa UANL-001L is growing on mineral medium, while an increase of $22 \%$ is observed when YM medium is used.

On the other hand, superficial tension decreases almost linearly with increasing concentrations of EOPO. In systems with mineral medium and no microorganisms, superficial tension only decreases from 1.47 to $3.44 \%$, while it decreases from $10 \%$ to $14.3 \%$ in systems with YM medium and no microorganisms. However, superficial tension is higher in systems comprised of mineral medium than in systems with YM medium. When Rhodotorula mucilaginosa UANL-001L is present on the system, superficial tension changes significantly only with YM medium. 
Contrary to the superficial tension behavior, the interfacial tension increases with an increase in EOPO concentration. The systems with YM medium present lower interfacial tension than the systems with mineral medium. However, interfacial tension measurements showed that, liquid-liquid phase formation (immiscible system) do not take place with EOPO concentration below 20\%, therefore, interfacial tension measurement was not possible. Consequently, extractive fermentation could not take place at concentrations below $20 \%$ EOPO concentration since immiscible phases are not formed.

Table 4. Two-phase system property measurements.

\begin{tabular}{|c|c|c|c|c|c|}
\hline \multirow[t]{2}{*}{ Medium } & EOPO 970 & Microorganism & Viscosity & $\begin{array}{l}\text { Superficial } \\
\text { Tension }(\sigma)\end{array}$ & $\begin{array}{c}\text { Interfacial } \\
\text { Tension }(\gamma)\end{array}$ \\
\hline & {$[\%$ o $v / v]$} & (Yes/No) & {$[\mathrm{Pa} \cdot \mathrm{s}]$} & {$[\mathrm{N} / \mathrm{m}]$} & {$[\mathrm{N} / \mathrm{m}]$} \\
\hline Mineral & 10 & No & 0.00227 & 0.0680 & ND \\
\hline Mineral & 20 & No & 0.00285 & 0.0680 & 0.0337 \\
\hline Mineral & 30 & No & 0.00329 & 0.0680 & 0.0367 \\
\hline Mineral & 40 & No & 0.00378 & 0.0670 & 0.0380 \\
\hline Mineral & 50 & No & 0.00422 & 0.0670 & 0.0387 \\
\hline Mineral & 0 & Yes & 0.00135 & 0.0677 & ND \\
\hline Mineral & 10 & Yes & 0.00237 & 0.0680 & ND \\
\hline Mineral & 20 & Yes & 0.00422 & 0.0670 & 0.0310 \\
\hline Mineral & 30 & Yes & 0.00605 & 0.0660 & 0.0330 \\
\hline Mineral & 40 & Yes & 0.00771 & 0.0660 & 0.0350 \\
\hline Mineral & 50 & Yes & 0.00987 & 0.0653 & 0.0367 \\
\hline YM & 10 & No & 0.00291 & 0.0467 & ND \\
\hline YM & 20 & No & 0.00385 & 0.0443 & ND \\
\hline YM & 30 & No & 0.00490 & 0.0430 & 0.0300 \\
\hline YM & 40 & No & 0.00696 & 0.0420 & 0.0317 \\
\hline YM & 50 & No & 0.00835 & 0.0420 & 0.0350 \\
\hline YM & 0 & Yes & 0.00168 & 0.0537 & ND \\
\hline YM & 10 & Yes & 0.00426 & 0.0500 & ND \\
\hline YM & 20 & Yes & 0.00510 & 0.0500 & 0.0280 \\
\hline YM & 30 & Yes & 0.00850 & 0.0490 & 0.0287 \\
\hline YM & 40 & Yes & 0.00841 & 0.0470 & 0.0300 \\
\hline $\mathrm{YM}$ & 50 & Yes & 0.01019 & 0.0460 & 0.0330 \\
\hline $\mathrm{YM}$ & 0 & No & 0.00154 & 0.0403 & - \\
\hline EOPO & 100 & No & 0.11363 & 0.0547 & - \\
\hline
\end{tabular}

\subsubsection{Two-Phase Generation in the Prescence of EOPO 970}

Phase formation time and temperature was studied by heating the cultures with different EOPO 970 concentration $(20,30,40$, and $50 \% v / v)$ in a water bath until reaching $30,35,40$, and $45^{\circ} \mathrm{C}$ culture temperature, and recording the time needed at each temperature to visualize phase formation. In Figure 4, the results of such analysis are depicted, where it is clear that, at higher temperatures, lower times are needed for phase formation regardless EOPO concentration in the culture. However, it is clear that higher EOPO concentrations require less time and temperature for phase formation, being at $40 \%$ and 50\% EOPO the most attractive alternatives, where 4 to $6 \mathrm{~min}$ are needed in order to observe phase formation at 30 to $45^{\circ} \mathrm{C}$. More importantly, after 6 min of phase formation time, higher temperatures do not provide conditions for significantly lower formation times in cultures with $40 \%$ and $50 \%$ EOPO concentration. 


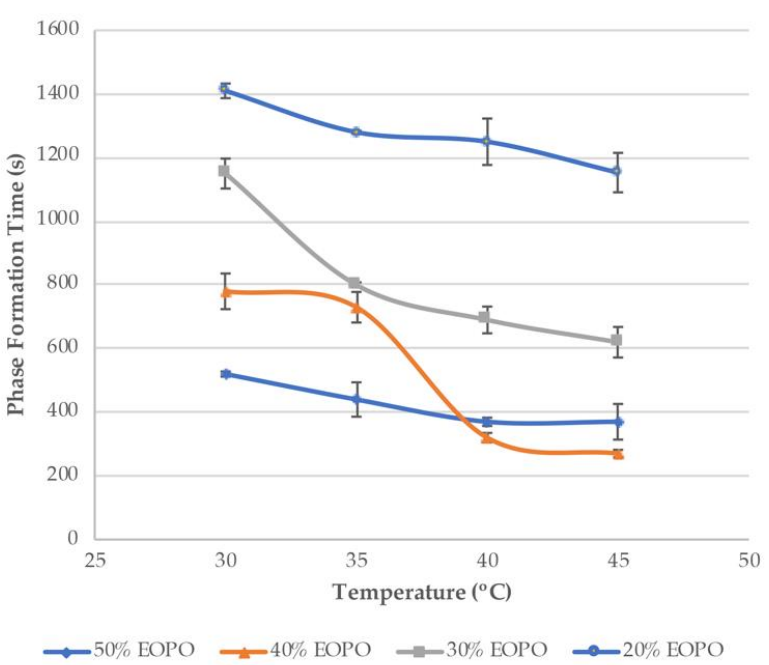

Figure 4. Phase formation time and temperature needed for different EOPO 970 concentrations (20, 30, 40 and $50 \% v / v$ ) on Rhodotorula mucilaginosa UANL-001L cultures in mineral medium.

\subsection{Biomass and EPS Production and Recovery on Extractive Fermentation with EOPO 970}

After phase formation with 20,30, 40, and 50\% v/v EOPO 970 concentration, each phase (bottom and top phase) was separated for biomass and EPS quantification. Figure 5 shows both biomass and EPS recovery \% for each separated phase. Cultures with 30, 40, and 50\% EOPO 970 concentration showed a biomass separation predominantly to the bottom phase with recoveries from $88 \%$ to $95 \%$, while EPS also partitioned predominantly to the bottom phase with recoveries from 37 to $45 \%$. It is important to note that cultures with $40 \%$ and $50 \%$ EOPO 970 provided the best conditions for biomass and EPS separation on different phases. For systems with $40 \%$ EOPO concentration, $7 \%$ of biomass and $42 \%$ of EPS is recovered on the top phase and $93 \%$ of biomass and $58 \%$ of EPS can be recovered in the bottom phase. For 50\% EOPO 970, a similar behavior was observed, since $5 \%$ of biomass and $37 \%$ of EPS is recovered on the top phase; while 95\% biomass and 63\% EPS can be recovered in the bottom phase. Although said systems do not provide conditions for full EPS and biomass partition to different phases, said EOPO 970 concentrations have potential for biomass and EPS separation on continuous flow extraction, since EPS could be recovered on the extracting phase and further separated with other methods.

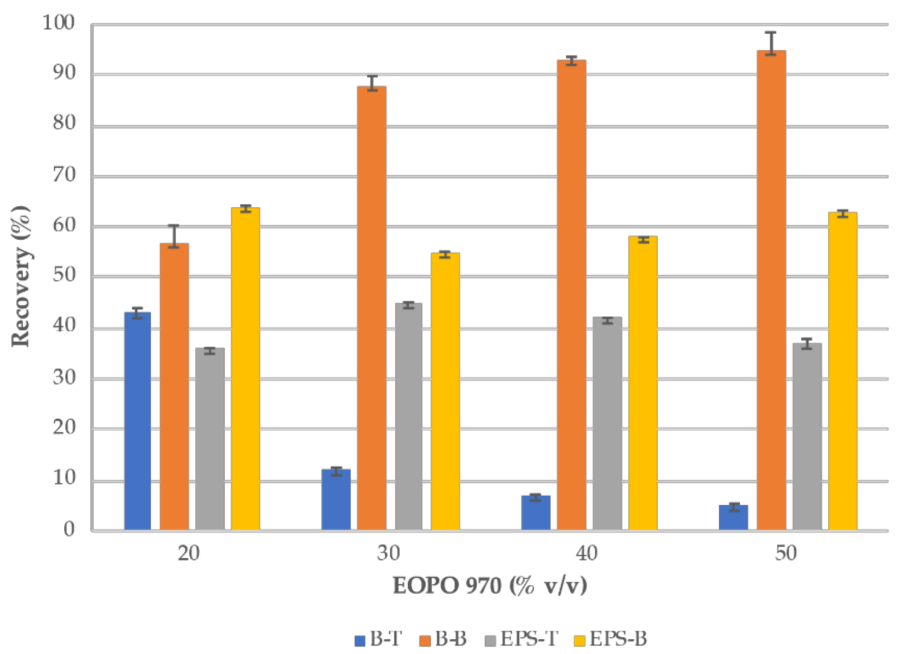

Figure 5. Rhodotorula mucilaginosa UANL-001L biomass (B) and exopolysaccharide (EPS) recovery in top (T) and bottom phase (B) of extractive fermentation with EOPO 970 at $35^{\circ} \mathrm{C}$. 


\section{Discussion}

Biomass and EPS production are highly affected by media composition and other culture conditions such as temperature, $\mathrm{pH}$, and agitation [12]. In the present study, both biomass and EPS generation was not significantly affected by media composition (glucose in YM medium vs saccharose in mineral medium), but biomass production resulted lower than the reported in different studies [13-16], where production reached up to $10.2 \mathrm{~g} / \mathrm{L}$ biomass concentration. However, it has also been reported that nitrogen content and source are important factors for biomass formation [17-19], and that yeast extract and ammonium nitrate are generally the best sources for yeast cultivation. For mineral medium, nitrogen was provided by ammonium chloride, so its substitution should be studied in order to determine if biomass production could be increased.

EPS production has been previously reported in other Rhodotorula strains, such as Rhodotorula mucilaginosa YR-2, where EPS production was observed after $120 \mathrm{~h}$ culture [20], which coincides with our results. However, EPS production has also been reported to be higher, since titters up to $7.8 \mathrm{~g} / \mathrm{L}$ have been reported [20]. Such difference on EPS production could be attributed to the low Rhodotorula mucilaginosa UANL-001L biomass concentrations obtained, as well as differences in the Rhodotorula mucilaginosa strain. More importantly, it has been reported that even on the same yeast strains, EPS production can result in different production rates or EPS composition, due to culture conditions and carbon source [21,22], so further investigation on culture conditions and carbon source is needed in order to obtain higher concentrations of EPS.

Since the aim of the present investigation was to apply extractive fermentation on the recuperation of EPS, it was of importance to first determine if EOPO added to the culture affected cell viability. EOPO is a polymer, therefore it adds viscosity to the medium, affecting the rheological attributes of the culture. Therefore, cultures with EOPO require higher mixing times [23], present lower oxygen diffusion due to coalescence [24] and a diminution of $\mathrm{pH}$ control [25], and microorganism metabolism rates [26]. According to Table 4, viscosity increases with increasing EOPO concentrations in both media, and that effect intensifies in mineral medium. Therefore, cell viability can be affected negatively due to the high culture viscosity when EOPO is used for extractive fermentation. In this scenario, Rhodotorula mucilaginosa UANL-001L biomass production was lower than the production previously reported for Burkholderia cepaci [27] in extractive fermentation with thermoseparating polymers, where polymer concentration reduced the microorganism growth rate. In other studies, polymers such as polyethylene glycol were proven to be toxic to E. coli depending on the polymer molecular weight and concentration, where lower molecular weights (200-600 MW) and concentrations (30\% v/v) resulted in lower cell viability losses (up to $20 \%$ loss) than higher molecular weights and concentrations (up to 70\% loss) [28].

Parameters such as viscosity, superficial tension, interfacial tension, phase formation time, and temperature for extractive fermentation are not frequently reported simultaneously. The most reported parameter in the literature is polymer concentration and temperature needed for phase formation in a 20 to $30 \mathrm{~min}$ time range $[27,29,30]$. More importantly, the temperature needed for phase formation is generally high $\left(30-50^{\circ} \mathrm{C}\right)$ in comparison to the optimal growth temperature for the microorganism, which can ultimately affect growth rates. For example, extractive fermentation implemented on Burkholderia cepaci, which grows in temperatures around $30{ }^{\circ} \mathrm{C}$, require a $50{ }^{\circ} \mathrm{C}$ temperature for $15 \mathrm{~min}$ in order to observe phase formation [27]. Said conditions can be of importance for not only for cell viability, but also for EPS production and recovery. In our study, lower temperatures were needed for EOPO to induce phase formation, which resulted in a range of 4 to 12 min needed for phase formation. Said conditions results in low differences between optimal growth temperature and phase formation temperature, which could be beneficial for Rhodotorula mucilaginosa UANL-001L in extractive fermentation. This is of importance, since phase formation temperatures should be compatible to ideal temperatures for microorganism growth, in order to not affect growth rates, production rates, oxygen diffusion and even production costs [26]. Moreover, phase formation temperatures should be short in order to maintain cell viability and cell culture stability [31]. 
Extractive fermentation has proven to be an adequate technique for the recovery and purification of different metabolites, such as enzymes [27,32], obtaining enzyme purity up to $90 \%$ applying temperatures high as $50{ }^{\circ} \mathrm{C}$ for $20 \mathrm{~min}$, and $87 \%$ purity with $65{ }^{\circ} \mathrm{C}$ for 15 min, respectively. However, since the products are of protein nature, it is hard to compare such operation conditions and purities obtained in said studies with our work. EPS is a complex high molecular weight product, therefore, its partition behavior, and conditions needed for its recovery and purification can be expected to be different than other products reported.

To our knowledge, biomass and EPS production and recovery in extractive fermentation has not been reported previously for any species of Rhodotorula. Recently, it was reported the implementation of extractive fermentation for the production of pullulan, an EPS produced by A. pullulans, in PEG-dextran or PEG-mono/bi-sodium systems [33]. This study reported that biomass was partitioned to the PEG rich top phase and that the pullulan was partitioned towards the bottom phase. In our study, biomass and EPS partition was dependent on EOPO concentration, where both products were partitioned predominantly to the bottom phase in various proportions. Although partition behavior is different for both microorganisms and extractive fermentation systems, it is clear that extractive fermentation can result in an important strategy for recovery and even purification of EPS.

Finally, we observed that extractive fermentation performance could be enhanced in a continuous flow extractor by taking advantage of the liquid-liquid system. Future work should be focused on the interaction between each extractive phase (EOPO + Medium + Microorganisms) to control mass transfer and provide operational conditions for a full EPS and biomass separation. Since interfacial tension increases with mineral medium and with EOPO concentration, experiments could be designed to favor different flow patterns, such as slug flow or drop flow, to increase the interaction between phases, and thus enhance the extraction and recovery.

\section{Conclusions}

Fermentation parameters such as EOPO concentration, and phase formation temperature and time were identified as first approaches to understand and determine the application of extractive fermentation for EPS production and recovery, with interesting results. This is the first report of extractive fermentation applied on EPS production and recovery from a yeast of the genera Rhodotorula, proving to be an interesting strategy, although further optimization is needed in order to increase biomass and EPS production by this technique. Since Rhodotorula mucilaginosa UANL-001L EPS is of special interest due to its bioadsorbent and wide spectrum antimicrobial properties, further study of extractive fermentation as a technique for production, recovery and purification is highly encouraged.

Author Contributions: Conceptualization, A.G.-L. and E.A.L.-G.; methodology, C.F.M.-R.; formal analysis, A.G.-L., E.A.L.-G. and C.F.M.-R.; investigation, C.F.M.-R., M.T.C.-G. and M.F.A.-G.; resources, A.M.-C. and J.R.M.-R.; writing-original draft preparation, C.F.M.-R., A.G.-L. and E.A.L.-G.; writing-review and editing, A.M.-C. and J.R.M.-R.; supervision, A.G.-L. and E.A.L.-G.; project administration, A.G.-L.; funding acquisition, A.G.-L. All authors have read and agreed to the published version of the manuscript.

Funding: This research was funded by CONACyT grant number 633454, Facultad de Ciencias Químicas de la Universidad Autónoma de Nuevo León and Programa para el Desarrollo Profesional Docente para el Tipo Superior (PRODEP) 2018.

Acknowledgments: Authors sincerely thanks to División de Estudios de Posgrado de la Facultad de Ciencias Químicas (UANL)

Conflicts of Interest: The authors declare no conflict of interest. The funders had no role in the design of the study; in the collection, analyses, or interpretation of data; in the writing of the manuscript, or in the decision to publish the results. 


\section{References}

1. Hussain, A.; Zia, K.M.; Tabasum, S.; Noreen, A.; Ali, M.; Iqbal, R.; Zuber, M. Blends and composites of exopolysaccharides; properties and applications: A review. Int. J. Biol. Macromol. 2017, 94, 10-27. [CrossRef] [PubMed]

2. Satyanarayana, T.; Gotthard, K. Yeast Biotechnology: Diversity and Applications, 1st ed.; Springer: New York, NY, USA, 2009. [CrossRef]

3. Andhare, P.; Chauhan, K.; Dave, M.; Pathak, H. microbial exopolysaccharides: Advances in applications and future prospects. In Biotechnology Volume 3: Microbial Biotechnology, 1st ed.; Tewari, R., Ed.; Studium Press LLC: Houston, TX, USA, 2013; Volume 3, pp. 1-26. [CrossRef]

4. Gonzalez Garza, M.T.; Barboza Perez, D.; Vazquez Rodriguez, A.; Garcia-Gutierrez, D.I.; Zarate, X.; Cantú Cardenas, M.E.; Urraca-Botello, L.I.; Lopez-Chuken, U.J.; Trevino-Torres, A.L.; Cerino-Córdoba, F.d.J.; et al. Metal-induced production of a novel bioadsorbent exopolysaccharide in a native Rhodotorula mucilaginosa from the mexican northeastern region. PLoS ONE 2016, 11, e0148430. [CrossRef]

5. Vazquez-Rodriguez, A.; Vasto-Anzaldo, X.; Barboza Perez, D.; Vázquez-Garza, E.; Chapoy-Villanueva, H.; García-Rivas, G.; Garza-Cervantes, J.; Gómez-Lugo, J.; Gomez-Loredo, A.; Garza Gonzalez, M.T.; et al. Microbial Competition of Rhodotorula mucilaginosa UANL-001L and E. coli increase biosynthesis of Non-Toxic Exopolysaccharide with Applications as a Wide-Spectrum Antimicrobial. Sci. Rep. 2018, 8, 1-14. [CrossRef] [PubMed]

6. Nwodo, U.U.; Green, E.; Okoh, A.I. Bacterial exopolysaccharides: Functionality and prospects. Int. J. Mol. Sci. 2012, 13, 14002-14015. [CrossRef] [PubMed]

7. Freitas, F.; Torres, C.A.V.; Reis, M.A.M. Engineering aspects of microbial exopolysaccharide production. Bioresour. Technol. 2017, 245, 1674-1763. [CrossRef] [PubMed]

8. Show, P.L.; Tan, P.C.; Anuar, M.S.; Ariff, A.; Yusof, Y.A.; Chen, S.K.; Ling, T.C. Direct recovery of lipase derived from Burkholderia Cepacia in recycling aqueous two-phase flotation. Sep. Purif. Technol. 2011, 80, 577-584. [CrossRef]

9. Zhu, J.H.; Yan, X.L.; Chen, H.J.; Wang, Z.H. In situ extraction of intracellular l-asparaginase using thermoseparating aqueous two-phase systems. J. Chromatogr. A 2007, 1147, 127-134. [CrossRef]

10. Raja, S.; Murty, V.R.; Varadavenkatesan, T.; Rajasekar, V.; Ramesh, V. Aqueous Two Phase Systems for the Recovery of Biomolecules-A Review. Sci. Technol. 2011, 1, 7-16. [CrossRef]

11. Rico-Castro, X.; González-Amado, M.; Soto, A.; Rodríguez, O. Aqueous two-phase systems with thermo-sensitive EOPO co-polymer (UCON) and sulfate salts: Effect of temperature and cation. J. Chem. Thermodyn. 2017, 108, 136-142. [CrossRef]

12. Ates, O. Systems biology of microbial exopolysaccharides production. Front. Bioeng. Biotechnol. 2015, 3, 1-16. [CrossRef]

13. Ghada, S.I.; Mahmoud, G.M.; Asker, M.; Eman, A.G. Production and biological evaluation of exopolysaccharide from isolated Rhodotorula glutinins. Aus. J. Basic Appl. Sci. 2012, 6, 401-408.

14. Sanchez, N.; Ramírez, D.; Zapata, A. evaluación de un sistema de fermentación extractiva para la producción de ácido láctico utilizando suero de leche como sustrato. VITAE Rev. Fac. Química Farm. 2007, 14, 27-34.

15. Pavlova, K.; Grigorova, D. Production and properties of exopolysaccharide by Rhodotorula acheniorum MC. Food Res. Int. 1999, 32, 473-477. [CrossRef]

16. Aksu, Z.; Tug, A. Carotenoids production by the yeast Rhodotorula mucilaginosa: Use of agricultural wastes as a carbon source. Process. Biochem. 2005, 40, 2985-2991. [CrossRef]

17. Ann, M.; Ramirez, J.R.; Dizon, E.I.; Mercado, S.M. Substrate optimization for exopolysaccharide production by Rhodotorula minuta BIOTECH 2178 using simplex-lattice design. J. Soc. Technol. 2015, 5, 34-41.

18. Chi, Z.; Zhao, S. Optimization of medium and cultivation conditions for pullulan production by a new pullulan-producing yeast strain. Enzyme Microb. Technol. 2003, 33, 206-211. [CrossRef]

19. Villay, A.; Laroche, C.; Roriz, D.; El Alaoui, H.; Delbac, F.; Michaud, P. Optimization of culture parameters for exopolysaccharides production by the microalga Rhodella violacea. Bioresour. Technol. 2013, 146, 732-735. [CrossRef]

20. Takita, J.; Itano, R.; Morii, N.; Ebina, F.; Matsuda, K.; Katohda, S. Production and rheology of exopolysaccharide by the yeast Rhodotorula mucilaginosa YR-2. J. Appl. Glycosci. 2004, 51, 255-257. [CrossRef] 
21. Yuksekdag, Z.N.; Aslim, B. Influence of different carbon sources on exopolysaccharide production by Lactobacillus delbrueckii subsp. bulgaricus (B3, G12) and Streptococcus thermophilus (W22). Braz. Arch. Biol. Technol. 2008, 51, 581-585. [CrossRef]

22. Grobben, G.J.; Smith, M.R.; Sikkema, J.; de Bont, J.A.M. Influence of fructose and glucose on the production of exopolysaccharides and the activities of enzymes involved in the sugar metabolism and the synthesis of sugar nucleotides in Lactobacillus delbrueckii subsp. bulgaricus NCFB 2772. Appl. Microbiol. Biotechnol. 1996, 46, 279-284. [CrossRef]

23. Schmid, J.; Sieber, V.; Rehm, B. Bacterial exopolysaccharides: Biosynthesis pathways and engineering strategies. Front. Microbiol. 2015, 6, 496. [CrossRef] [PubMed]

24. Kold, D. Study of Mass Transfer in Viscous Fermentations. Ph.D. Thesis, Technical University of Denmark, Lyngby, Denmark, 2010.

25. Schmidt, F.R. Optimization and scale up of industrial fermentation processes. Appl. Microbiol. Biotechnol. 2005, 68, 425-435. [CrossRef] [PubMed]

26. Humbird, D.; Fei, Q. Scale-Up Considerations for Biofuels. In Biotechnology for Biofuel Production and Optimization, 1st ed.; Eckert, C.A., Trinh, C.T., Eds.; Elsevier: Amsterdam, The Netherlands, 2016; Volume 1, pp. 513-517. [CrossRef]

27. Show, P.L.; Tan, C.P.; Anuar, M.S.; Ariff, A.; Yusof, Y.A.; Chen, S.K.; Ling, T.C. Extractive fermentation for improved production and recovery of lipase derived from Burkholderia cepacia using a thermoseparating polymer in aqueous two-phase systems. Bioresour. Technol. 2012, 116, 226-233. [CrossRef] [PubMed]

28. Cox, C.S. Bacterial survival in suspension in polyethylene glycol solutions. J. Gen. Microbiol. 1966, 45, $275-281$. [CrossRef] [PubMed]

29. Chen, B.; Han, J.; Wang, Y.; Sheng, C.; Liu, Y.; Zhang, G.; Yan, Y. Separation, enrichment and determination of ciprofloxacin using thermoseparating polymer aqueous two-phase system combined with high performance liquid chromatography in milk, egg, and shrimp samples. Food Chem. 2014, 148, 105-111. [CrossRef] [PubMed]

30. Cheng, Z.; Song, H.; Cao, X.; Shen, Q.; Han, D.; Zhong, F.; Hu, H.; Yang, Y. Simultaneous extraction and purification of polysaccharides from Gentiana scabra Bunge by microwave-assisted ethanol-salt aqueous two-phase system. Ind. Crop. Prod. 2017, 102, 75-87. [CrossRef]

31. Prasad, P.V.; Pisipati, S.; Mutava, R.N.; Tuinstra, M. Sensitivity of grain sorghum to high temperature stress during reproductive development. Crop. Sci. 2008, 48, 1911-1917. [CrossRef]

32. Ng, H.S.; Tan, C.P.; Mokhtar, M.N.; Ibrahim, S.; Ariff, A.; Ooi, C.W.; Ling, T.C. Recovery of Bacillus cereus cyclodextrin glycosyltransferase and recycling of phase components in an aqueous two-phase system using thermo-separating polymer. Sep. Purif. Technol. 2012, 89, 9-15. [CrossRef]

33. Badhwar, P.; Kumar, P.; Kumar Dubey, K. Extractive fermentation for process integration and amplified pullulan production by A. pullulans in Aqueous Two Phase Systems. Sci. Rep. 2019, 9, 1-8. [CrossRef]

Publisher's Note: MDPI stays neutral with regard to jurisdictional claims in published maps and institutional affiliations.

(C) 2020 by the authors. Licensee MDPI, Basel, Switzerland. This article is an open access article distributed under the terms and conditions of the Creative Commons Attribution (CC BY) license (http://creativecommons.org/licenses/by/4.0/). 